\title{
Clinicopathologic and prognostic associations of KRAS and BRAF mutations in small intestinal adenocarcinoma
}

\author{
Sun-Young Jun ${ }^{1}$, Misung $\mathrm{Kim}^{2}$, Mi Jin $\mathrm{Gu}^{3}$, Young Kyung Bae ${ }^{3}$, Hee-Kyung Chang ${ }^{4}$, \\ Eun Sun Jung ${ }^{5}$, Kee-Taek Jang ${ }^{6}$, Jihun Kim ${ }^{7}$, Eunsil Yu ${ }^{7}$, Dae Woon Eom ${ }^{8,9}$ and \\ Seung-Mo Hong 7,9
}

${ }^{1}$ Department of Pathology, Incheon St. Mary's Hospital, The Catholic University of Korea, Incheon, Republic of Korea: ${ }^{2}$ Department of Pathology, Ulsan University Hospital, University of Ulsan College of Medicine, Ulsan, Republic of Korea; ${ }^{3}$ Department of Pathology, Yeungnam University College of Medicine, Daegu, Republic of Korea; ${ }^{4}$ Department of Pathology, Kosin University College of Medicine, Busan, Republic of Korea; ${ }^{5}$ Department of Pathology, The Catholic University of Korea College of Medicine, Seoul, Republic of Korea; ${ }^{6}$ Department of Pathology, Samsung Medical Center, Sungkyunkwan University School of Medicine, Seoul, Republic of Korea; ${ }^{7}$ Department of Pathology, Asan Medical Center, University of Ulsan College of Medicine, Seoul, Republic of Korea and ${ }^{8}$ Department of Pathology, Gangneung Asan Hospital, University of Ulsan College of Medicine, Gangneung, Republic of Korea

\begin{abstract}
Activating $K R A S$ and/or BRAF mutations have been identified as predictors of resistance to anti-epidermal growth factor receptor (EGFR) chemotherapy in colorectal cancer. But the status of KRAS and BRAF mutations and their clinicopathologic and prognostic significance has not been extensively evaluated in small intestinal adenocarcinomas. In this work, the KRAS and BRAF genes in 190 surgically resected small intestinal adenocarcinoma cases were sequenced and their association with various clinicopathologic variables, including survival of the patients, was analyzed. KRAS or BRAF mutations were observed in $63(33 \%)$ cases. Sixty-one cases had KRAS mutations and 2 had BRAF mutations and the two types of mutation were mutually exclusive. The majority of KRAS mutations were $G>A$ transition $(43 / 61$ cases, $71 \%)$ or p.G12D (31/61 cases, $51 \%)$. The patients with mutant KRAS tended to have higher pT classifications $(P=0.034)$ and more frequent pancreatic invasion $(P=0.020)$ than those with wild-type KRAS. Multivariate logistic regression analysis showed that certain mutated KRAS subtypes ( $\mathrm{G}>\mathrm{A}$ transitions and $\mathrm{G12D}$ mutations) were significantly correlated with higher $\mathrm{pT}$ classification ( $P=0.015$ and 0.004 , respectively) than wild-type KRAS and other KRAS mutations. The patients with KRAS or BRAF mutation had a tendency to shorter overall survival than those with wild-type KRAS and $B R A F(P=0.148)$, but subgroup analysis demonstrated the patients with KRAS mutations showed worse survival (median, 46.0 months; $P=0.046$ ) than those with wild-type KRAS (85.4 months) in lower pT classification (pT1pT3) group. In summary, KRAS and, infrequently, BRAF mutations are observed in a subset of small intestinal adenocarcinomas, and are associated with higher pT classification and more frequent pancreatic invasion. KRAS mutation is a poor prognostic predictor in patients with lower pT classification tumors. Anti-EGFR targeted therapy could be applied to about two-thirds of small intestinal adenocarcinoma patients, namely those with wildtype KRAS and BRAF if they have metastatic disease, similar to colorectal cancer patients.
\end{abstract}

Modern Pathology (2016) 29, 402-415; doi:10.1038/modpathol.2016.40; published online 19 February 2016

Correspondence: Dr DW Eom, MD, PhD, Department of Pathology, Gangneung Asan Hospital, University of Ulsan College of Medicine, 415, Bangdong-ri, Sacheon-myeon, Gangneung, Gangwon-do 210-711, Republic of Korea or Professor S-M Hong, $\mathrm{MD}$, PhD, Department of Pathology, Asan Medical Center, University of Ulsan College of Medicine, 88, Olympic-ro 43-gil, Songpa-gu, Seoul 138-736, Republic of Korea.

E-mail: edwjyh@gnah.co.kr or smhong28@gmail.com

${ }^{9}$ These authors contributed equally to this work.

Received 29 November 2015; revised 7 January 2015; accepted 7 January 2016; published online 19 February 2016
Although the small intestine accounts for $90 \%$ of the mucosal surface area of the gastrointestinal tract, small intestinal adenocarcinoma is rare and accounts for only approximately $2 \%$ of gastrointestinal malignancies. ${ }^{1}$ Epidemiologic observations have shown an increasing incidence of small intestinal adenocarcinoma in recent years, with an estimated 9160 new cases in 2014 in the United States. ${ }^{2}$

Small intestinal adenocarcinoma has a poor prognosis at all stages, which appears to be intermediate 
between that of colon and gastric cancer. ${ }^{3}$ Lymph node metastasis and distal location (jejunum and ileum) are the most important independent prognostic factors. ${ }^{4}$ As a result of the nonspecific nature of presenting clinical manifestations and the lack of effective tools for exploring the small intestine, small intestinal adenocarcinomas are usually diagnosed at an advanced stage ${ }^{5}$ at which chemotherapy can only prolong overall survival. The cytotoxic agents used are generally the same as those used to treat patients with advanced colorectal cancers, and include oral or intravenous fluoropyrimidines, platinum and irinotecan. ${ }^{5}$ Unfortunately, there is no standard treatment and the place of new targeted therapies has still to be defined. ${ }^{1}$ Moreover, the number of tumor samples collected remains limited because of the low incidence of the disease, which explains the lack of information regarding somatic genetic alterations that occur during small intestinal carcinogenesis.

The RAS-RAF mitogen-activated protein kinase cascade has a crucial role in tumor cell proliferation. The KRAS oncogene is mutated in approximately 30$40 \%$ of colorectal cancers, mainly at codon 12 or 13 . KRAS mutations are predictive of a lack of efficacy of anti-epidermal growth factor receptor (EGFR) monoclonal antibodies, such as panitumumab and cetuximab, in patients with metastatic colorectal cancer. ${ }^{6}$ Recently, several case reports have described the effect of anti-EGFR therapy in patients with wild-type KRAS small intestinal adenocarcinomas. ${ }^{7,8}$ Moreover, a number of studies of combined treatments, such as CAPOX (capecitabine and oxaliplatin) with bevacizumab, CAPOX with irinotecan, CAPOX with panitumumab, GEMOX (gemcitabine and oxaliplatin) with erlotinib and Nab-paclitaxel, are exploring the effects of anti-EGFR agents in small intestinal adenocarcinoma. ${ }^{9}$ BRAF mutations, which always occur in the absence of KRAS mutations, have also been associated with resistance to anti-EGFR treatment in colorectal cancers.

$K R A S$ and $B R A F$ are two of the most frequently studied oncogenes, but they have been little studied in small intestinal adenocarcinoma. ${ }^{1,3,10-20}$ In addition, because most of these few analyses were performed on only small numbers of small intestinal adenocarcinoma patients, they need to be validated.

In this study, we analyze the mutational status of KRAS and BRAF in small intestinal adenocarcinomas and consider the utility of targeted therapy in the light of their frequency. In addition, we evaluate their clinicopathologic and prognostic significance.

\section{Materials and methods}

\section{Study Population}

This study was approved by the Institutional Review Board of Incheon St. Mary's Hospital (OC14OIMI0133). A total cohort of 197 surgically resected primary small intestinal adenocarcinoma cases was collected from the surgical pathology archives of 22 South Korean institutions by the Korean Small Intestinal Cancer Study Group, as previously reported. ${ }^{21}$ Carcinomas extending from the surrounding gastrointestinal tract organs, such as the stomach, ampulla of Vater, pancreas, cecum or appendix, into the small bowel were excluded from the analysis. Clinical and pathologic data collected as part of a previous study were used again in this study. ${ }^{21}$

Histologic types and tumor grading were classified according to the WHO classification. ${ }^{22}$ Briefly, tumor grading was classified as low-grade (well ( $>95 \%$ with gland formation) and moderately differentiated (50-95\% with gland formation) adenocarcinomas) and high-grade (poorly differentiated (1-49\% with gland formation) and undifferentiated (no gland formation)) carcinomas. ${ }^{22}$

\section{Molecular Analysis}

Ten sections with $10 \mu \mathrm{m}$ in thickness from formalinfixed paraffin-embedded tissue blocks were used and manually dissected to extract genomic DNA. Genomic DNA was extracted with a QIAmp DNA Mini Kit (Qiagen, Valencia, CA, USA) following the manufacturer's protocol. Mutations in codons 12 and 13 of KRAS exon 1 and codon 600 of BRAF exon 15 were identified by cycle sequencing. The KRAS and BRAF genes were PCR amplified with primers for KRAS (F: 5'-TGACATGTTCTAATATAGTCAC-3', R: 5'-AC AAGATTTACCTCTATTGTT-3') and primers for BRAF (F: 5'-TCATAATGCTTGCTCTGATAGGA-3', R: 5'-GGCCAAAAATTTAATCAGTGGA-3'). In general, PCR reactions were run in a total volume of $25 \mu \mathrm{l}$ with $0.3 \mu \mathrm{M}$ of each primer using AmpliTaq Gold PCR Master Mix (Applied Biosystems, Foster City, CA, USA). Samples were subjected to initial denaturation at $95{ }^{\circ} \mathrm{C}$ for $15 \mathrm{~min}, 40-45$ cycles at $95^{\circ} \mathrm{C}$ for $50 \mathrm{~s}$, annealing for $50 \mathrm{~s}$ and elongation at $72{ }^{\circ} \mathrm{C}$ for $1 \mathrm{~min}$, followed by final elongation at $72^{\circ} \mathrm{C}$ for $7 \mathrm{~min}$. PCR products were column-purified using a QIAquick PCR Purification Kit (Qiagen) or enzymatically treated with ExoSAP-IT (USB, Cleveland, OH, USA). The sequencing primers were identical to the PCR primers, and all samples were sequenced in both directions using a BigDye Terminator Cycle Sequencing Kit, version 1.1 (Applied Biosystems). The sequencing reactions were analyzed on an ABI Prism 3100 Genetic Analyzer with Sequencing Analysis software, version 3.7 (Applied Biosystems).

\section{Statistical Analysis}

Statistical analyses were performed using SPSS software (version 17.0; SPSS, Chicago, IL, USA). Student's $t$-test, the $\chi^{2}$ test and Fisher's exact test were used to examine associations between categorical variables. Multivariate relationships were estimated by fitting logistic regression models. Overall patient survival was defined as the time from surgical 
Table 1 KRAS mutation status among small intestinal adenocarcinomas

\begin{tabular}{|c|c|c|c|c|c|}
\hline \multirow[b]{2}{*}{ KRAS mutation } & \multirow[b]{2}{*}{ Amino acid } & \multirow[b]{2}{*}{$\mathrm{n}$} & \multicolumn{2}{|c|}{ Tumor location (\%) } & \multirow[b]{2}{*}{ P-value } \\
\hline & & & Proximal (duodenum) & Distal (jejunum, ileum) & \\
\hline Transition of base & & 61 & $38(62)$ & $23(38)$ & $0.038^{\mathrm{a}}$ \\
\hline $\mathrm{G}>\mathrm{A}$ & & 43 & $26(60)$ & $17(40)$ & \\
\hline $\mathrm{G}>\mathrm{C}$ & & 5 & $1(20)$ & $4(80)$ & \\
\hline $\mathrm{G}>\mathrm{T}$ & & 13 & $11(85)$ & $2(15)$ & \\
\hline Mutation sequence & & & & & $0.042^{\mathrm{a}}$ \\
\hline Codon 12 & & 50 & $31(62)$ & $19(38)$ & \\
\hline GGT $>$ GCT & G12A (alanine) & 4 & 0 & $4(100)$ & \\
\hline GGT $>$ GTT & G12V (valine) & 6 & $6(100)$ & 0 & \\
\hline GGT > CGT & G12R (arginine) & 1 & $1(100)$ & 0 & \\
\hline GGT $>$ GAT & G12D (aspartate) & 31 & $18(58)$ & $13(42)$ & \\
\hline GGT $>$ TGT & G12C (cysteine) & 7 & $5(71)$ & $2(29)$ & \\
\hline GGT $>$ AGT & G12S (serine) & 1 & $1(100)$ & 0 & \\
\hline Codon 13 & & 11 & $7(64)$ & $4(36)$ & \\
\hline $\mathrm{GGC}>\mathrm{GAC}$ & G13D (aspartate) & 11 & $7(64)$ & $4(36)$ & \\
\hline
\end{tabular}

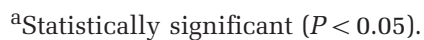

resection to death or last follow-up examination. Survival rates were calculated by the Kaplan-Meier method. Associations between survival rates and various clinicopathologic factors were assessed using the log-rank test. We also investigated the significance of any prognostic factors using Cox proportional hazards modeling. $P$-values $<0.05$ were considered to denote statistical significance.

\section{Results}

\section{Patient Characteristics}

Of the total of 197 patients, 190 with interpretable $K R A S$ or $B R A F$ sequencing results were included in our study. The mutation status of KRAS and BRAF was evaluable in 190 and 178 patients, respectively. We could not perform complete sequencing on the other samples because of a lack DNA or degradation of the DNA.

Patient ages ranged between 23 and 86 years (mean, 59.0 years; s.d., 14.0 years). The male to female ratio was 1.7. The median follow-up period after surgical resection was 28.6 months (range, 0-168.4 months). Predisposing conditions were observed in 22 cases $(12 \%)$, including 16 cases of sporadic adenoma, 3 of Peutz-Jeghers syndrome, 2 of Meckel's diverticulum and 1 of Crohn's disease. Forty patients were classified as 'suspected Lynch syndrome' based on revised Bethesda guideline. There were no patients with familial adenomatous polyposis, Gardner syndrome, gluten-sensitive enteropathy or intestinal duplication. Tumors were located in the duodenum in 105 cases (55\%), the jejunum in 56 cases $(30 \%)$ and the ileum in 29 cases $(15 \%)$.

\section{KRAS and BRAF Mutations}

KRAS mutations were observed in 32\% (61/190 cases) of the small intestinal adenocarcinoma patients, whereas $B R A F$ mutation was detected in only $1 \% \quad(2 / 178$ cases). The KRAS and BRAF mutations were mutually exclusive.

The KRAS mutations were mostly in codon 12 (Table 1). Fifty patients (50/61 cases, $82 \%$ ) had KRAS mutations in codon 12 , and 11 patients (11/61 cases, $18 \%$ ) had KRAS mutations in codon 13. Based on the mutation sequence of $K R A S$, the most frequent KRAS mutation subtype-expressed in the recommended genetic nomenclature ${ }^{23}$ - was p.G12D (31/61 cases, 51\%), followed by p.G13D (11/61, 18\%), p.G12C (7/61, 11\%), p.G12V (6/61, 10\%), p. G12A (4/61, 6\%), p.G12R (1/61, 2\%) and p.G12S $(1 / 61,2 \%)$. There was a significant difference in the mutation sequences of KRAS according to tumor location $(P=0.042)$ : p.G12D, p.G12C and p.G13D were more common in the proximal (duodenal) adenocarcinomas. p.G12A was detected only in distal adenocarcinomas, whereas p.G12V, p.G12R and p.G12S were found only in proximal adenocarcinomas. The most common base substitution in the KRAS mutations was a $\mathrm{G}$ to $\mathrm{A}$ transition $(\mathrm{G}>\mathrm{A}$; 43/61 cases, $71 \%)$, followed by $\mathrm{G}>\mathrm{T}(13 / 61,21 \%)$ and $\mathrm{G}>\mathrm{C}(5 / 61,8 \%)$. An association between the type of base substitution and tumor location was also observed $(P=0.038): \mathrm{G}>\mathrm{A}$ and $\mathrm{G}>\mathrm{T}$ substitutions were common in the proximal small intestine, whereas $\mathrm{G}>\mathrm{C}$ was common in the distal small intestine.

\section{The Association Between KRAS or BRAF Mutations and Clinicopathologic Factors}

The associations between KRAS mutation and clinicopathologic factors are summarized in Table 2. The patients with mutant KRAS tended to have higher $\mathrm{T}$ classifications $(P=0.034)$ and more frequent pancreatic invasion $(P=0.020)$ than those with wild-type KRAS. However, high-grade carcinomas were more common among adenocarcinomas 
with wild-type KRAS $(P=0.036)$. There was no association between KRAS mutation and other clinicopathologic factors, including age and gender, growth pattern, tumor location, histologic type, nodal metastasis, other loop invasion, retroperitoneal seeding, and perineural and lymphovascular invasion.

Similarly, patients with mutant KRAS or $B R A F$ had higher $\mathrm{T}$ classification $(P=0.040)$ and more frequent pancreatic invasion $(P=0.017)$, whereas high-grade carcinomas were more frequent in adenocarcinomas with wild-type $K R A S$ and $B R A F$ $(P=0.024)$.

\section{Association Between KRAS Mutation and Clinicopathologic Factors According to Tumor Location}

We analyzed in detail the relationship between pathologic factors and KRAS mutation according to tumor location (Table 3). Proximal adenocarcinoma patients with $K R A S$ mutations tended to have tumors with high $\mathrm{T}$ classifications $(P=0.022)$ and more frequent pancreatic invasion $(P=0.045)$, whereas distal cases with KRAS mutations had lower-grade tumors $(P=0.028)$.

We next evaluated the relationship between pathologic factors and KRAS mutation subtypes according to tumor location (Tables 4 and 5). In the proximal adenocarcinomas, KRAS G>A and G12D mutations were closely associated with late $\mathrm{T}$ classification $(P=0.015$ and 0.009 , respectively) and pancreatic invasion $(P=0.011$ and 0.003, respectively). By contrast, neither KRAS $\mathrm{G}>\mathrm{A}$ nor G12D mutations were associated with any pathologic factors among the distal adenocarcinomas.

\section{Association Between Subtypes of KRAS Mutations and Clinicopathologic Factors}

We assessed the correlation of KRAS mutational subtype with clinicopathologic variables (Tables 6 and 7). In a univariate association analysis stratified by KRAS mutation subtype, KRAS G>A mutation was significantly more frequent in tumors with a higher $\mathrm{T}$ classification $(P=0.015)$ and pancreatic invasion $(P=0.017)$ than in KRAS wild-type tumors and those with mutations other than $G>A$. The KRAS G>A mutation was not related to tumor location or differentiation status. In a multivariate logistic regression analysis including location, differentiation, $\mathrm{T}$ stage and pancreatic invasion, the KRAS G>A mutation remained significantly more frequent in late $\mathrm{T}$ stage tumors (odds ratio $=1.588$, 95\% CI: $1.085-2.324 ; P=0.017)$.

In a univariate analysis stratified by KRAS mutation subtype, the KRAS G12D mutation was more closely related to higher $\mathrm{T}$ classification $(P=0.004)$ and pancreatic invasion $(P=0.016)$ than the $K R A S$ wild-type and mutations other than G12D. In a
Table 2 Correlation between clinicopathological factors and KRAS mutation status in small intestinal adenocarcinomas

\begin{tabular}{|c|c|c|c|}
\hline \multirow[b]{2}{*}{ Clinicopathologic factor } & \multicolumn{2}{|c|}{ KRAS status (\%) } & \multirow[b]{2}{*}{ P-value } \\
\hline & Wild & Mutated & \\
\hline No. of patients & 129 & 61 & \\
\hline Size (mean \pm s.d., cm) & $4.4 \pm 2.6^{\mathrm{a}}$ & $4.5 \pm 2.2$ & 0.868 \\
\hline \multicolumn{4}{|l|}{ Age } \\
\hline$\leq 50$ & $38(29)$ & $15(25)$ & \multirow[t]{2}{*}{0.485} \\
\hline$>50$ & $91(71)$ & $46(75)$ & \\
\hline \multicolumn{4}{|l|}{ Sex } \\
\hline Male & $83(64)$ & $36(59)$ & \multirow[t]{2}{*}{0.479} \\
\hline Female & $46(36)$ & $25(41)$ & \\
\hline \multicolumn{4}{|l|}{ Growth pattern ${ }^{\mathrm{a}}$} \\
\hline Polypoid & $24(20)$ & $11(18)$ & \multirow{3}{*}{0.885} \\
\hline Nodular & $8(6)$ & $3(5)$ & \\
\hline Infiltrative & $90(74)$ & $46(77)$ & \\
\hline \multicolumn{4}{|l|}{ Location } \\
\hline Proximal (duodenum) & $67(52)$ & $38(62)$ & \multirow{2}{*}{0.180} \\
\hline Distal (jejunum, ileum) & $62(48)$ & $23(38)$ & \\
\hline \multicolumn{4}{|l|}{ Histologic subtype } \\
\hline Tubular & $113(88)$ & $59(96)$ & \multirow[t]{4}{*}{0.288} \\
\hline Mucinous & $8(6)$ & $1(2)$ & \\
\hline Signet ring cell & $4(3)$ & 0 & \\
\hline Undifferentiated & $4(3)$ & $1(2)$ & \\
\hline \multicolumn{4}{|l|}{ Differentiation } \\
\hline Low & $92(71)$ & $52(85)$ & \multirow{2}{*}{$0.036^{\mathrm{b}}$} \\
\hline High & $37(29)$ & $9(15)$ & \\
\hline \multicolumn{4}{|l|}{ pT classification ${ }^{\mathrm{c}}$} \\
\hline pT1-pT3 & $61(48)$ & $18(31)$ & \multirow[t]{2}{*}{$0.034^{\mathrm{b}}$} \\
\hline pT4 & $67(52)$ & 40 (69) & \\
\hline \multicolumn{4}{|l|}{$p N$ classification ${ }^{\mathrm{a}}$} \\
\hline No & $55(48)$ & $29(50)$ & \multirow[t]{2}{*}{0.787} \\
\hline N1 & $60(52)$ & $29(50)$ & \\
\hline \multicolumn{4}{|l|}{ Pancreas invasion } \\
\hline No & $90(70)$ & $32(52)$ & \multirow[t]{2}{*}{$0.020^{\mathrm{b}}$} \\
\hline Yes & $39(30)$ & $29(48)$ & \\
\hline \multicolumn{4}{|l|}{ Other loop invasion } \\
\hline No & $125(97)$ & 60 (98) & \multirow[t]{2}{*}{1.000} \\
\hline Yes & $4(3)$ & $1(2)$ & \\
\hline \multicolumn{4}{|l|}{ Retroperitoneal seeding } \\
\hline No & $121(94)$ & $57(93)$ & 1.000 \\
\hline Yes & $8(6)$ & $4(7)$ & \\
\hline Perineural invasion & & & \\
\hline No & $88(68)$ & 42 (69) & 0.930 \\
\hline Yes & $41(32)$ & $19(31)$ & \\
\hline Lymphovascular invasion & & & \\
\hline No & $61(47)$ & $30(49)$ & 0.807 \\
\hline Yes & $68(53)$ & $31(51)$ & \\
\hline
\end{tabular}

Abbreviation: s.d., standard deviation.

${ }^{\mathrm{a} C}$ Calculated using only patients with adequate data.

${ }^{\mathrm{b}}$ Statistically significant $(P<0.05)$.

${ }^{\mathrm{C}}$ Excluding patients with pTis. 
Table 3 Correlation between clinicopathologic features and KRAS mutation status according to tumor location

\begin{tabular}{|c|c|c|c|c|c|}
\hline & \multicolumn{2}{|c|}{ Proximal (duodenum) (\%) } & \multicolumn{3}{|c|}{ Distal (jejunum, ileum) (\%) } \\
\hline & Wild & Mutated & Wild & & Mutated \\
\hline Size (mean \pm s.d., $\mathrm{cm})$ & $4.2 \pm 2.5$ & $4.6 \pm 2.3$ & $4.6 \pm 2.8^{\mathrm{a}}$ & & $4.2 \pm 2.1$ \\
\hline$P$-value & \multicolumn{2}{|c|}{0.394} & \multicolumn{3}{|c|}{0.512} \\
\hline \multicolumn{6}{|l|}{ Age } \\
\hline$\leq 50$ & $20(30)$ & $9(24)$ & $18(29)$ & & $6(26)$ \\
\hline$>50$ & $47(70)$ & $29(76)$ & $44(71)$ & & $17(74)$ \\
\hline$P$-value & \multicolumn{2}{|c|}{0.497} & \multicolumn{3}{|c|}{0.789} \\
\hline \multicolumn{6}{|l|}{ Sex } \\
\hline Male & $43(64)$ & $23(61)$ & $40(65)$ & & $13(57)$ \\
\hline Female & $24(36)$ & $15(39)$ & $22(35)$ & & $10(43)$ \\
\hline$P$-value & \multicolumn{2}{|c|}{0.710} & \multicolumn{3}{|c|}{0.499} \\
\hline \multicolumn{6}{|l|}{ Growth pattern ${ }^{\mathrm{a}}$} \\
\hline Polypoid & $10(17)$ & $8(22)$ & $14(23)$ & & $3(13)$ \\
\hline Nodular & $4(6)$ & $2(5)$ & $4(6)$ & & $1(4)$ \\
\hline Infiltrative & $46(77)$ & $27(73)$ & $44(71)$ & & $19(83)$ \\
\hline$P$-value & \multicolumn{2}{|c|}{0.867} & \multicolumn{3}{|c|}{0.620} \\
\hline \multicolumn{6}{|l|}{ Histologic subtype } \\
\hline Tubular & $59(88)$ & 37 (97) & $54(87)$ & & $22(96)$ \\
\hline Mucinous & $4(6)$ & $1(3)$ & $4(6)$ & & 0 \\
\hline Signet ring cell & $3(4)$ & 0 & $1(2)$ & & 0 \\
\hline Undifferentiated & $1(2)$ & 0 & $3(5)$ & & $1(4)$ \\
\hline$P$-value & \multicolumn{2}{|c|}{0.489} & \multicolumn{3}{|c|}{0.866} \\
\hline \multicolumn{6}{|l|}{ Differentiation } \\
\hline Low & $50(75)$ & $31(82)$ & $42(68)$ & & $21(91)$ \\
\hline High & $17(25)$ & 7 (18) & $20(32)$ & & $2(9)$ \\
\hline$P$-value & \multicolumn{2}{|c|}{0.415} & \multicolumn{3}{|c|}{$0.028^{\mathrm{b}}$} \\
\hline$p T$ classification $^{\mathrm{C}}$ & & & & & \\
\hline pT1-pT3 & $26(39)$ & $6(17)$ & $35(56)$ & & $12(52)$ \\
\hline pT4 & $40(61)$ & $29(83)$ & $27(44)$ & & $11(48)$ \\
\hline$P$-value & & & & 0.725 & \\
\hline$p N$ classification ${ }^{\mathrm{a}}$ & & & & & \\
\hline No & $31(48)$ & $18(49)$ & $24(48)$ & & $11(52)$ \\
\hline N1 & $34(52)$ & $19(51)$ & $26(52)$ & & $10(48)$ \\
\hline$P$-value & & & & 0.736 & \\
\hline Pancreas invasion & & & & & \\
\hline No & $29(43)$ & $9(24)$ & $61(98)$ & & $23(100)$ \\
\hline Yes & $38(57)$ & $29(76)$ & $1(2)$ & & 0 \\
\hline$P$-value & & & & 1.000 & \\
\hline Other loop invasion & & & & & \\
\hline No & $67(100)$ & $37(97)$ & $58(94)$ & & $23(100)$ \\
\hline Yes & 0 & $1(3)$ & $4(6)$ & & 0 \\
\hline$P$-value & & & & 0.570 & \\
\hline Retroperitoneal seedin & & & & & \\
\hline No & $66(99)$ & $38(100)$ & $55(89)$ & & $19(83)$ \\
\hline Yes & $1(1)$ & 0 & $7(11)$ & & $4(17)$ \\
\hline$P$-value & & & & 0.479 & \\
\hline Perineural invasion & & & & & \\
\hline No & $47(70)$ & $26(68)$ & $41(66)$ & & $16(70)$ \\
\hline Yes & $20(30)$ & $12(32)$ & $21(34)$ & & $7(30)$ \\
\hline$P$-value & & & & 0.765 & \\
\hline Lymphovascular invas & & & & & \\
\hline No & $33(49)$ & $20(53)$ & $28(45)$ & & $10(43)$ \\
\hline Yes & $34(51)$ & $18(47)$ & $34(55)$ & & $13(57)$ \\
\hline$P$-value & & & & 0.890 & \\
\hline
\end{tabular}

Abbreviation: s.d., standard deviation.

${ }^{\mathrm{a}}$ Calculated using only patients with sufficient available data.

${ }^{\mathrm{b}}$ Statistically significant $(P<0.05)$.

${ }^{\mathrm{C}}$ Excluding patients with pTis. 
Table 4 Correlation between clinicopathologic features and the presence of KRAS G $>$ A mutations according to tumor location

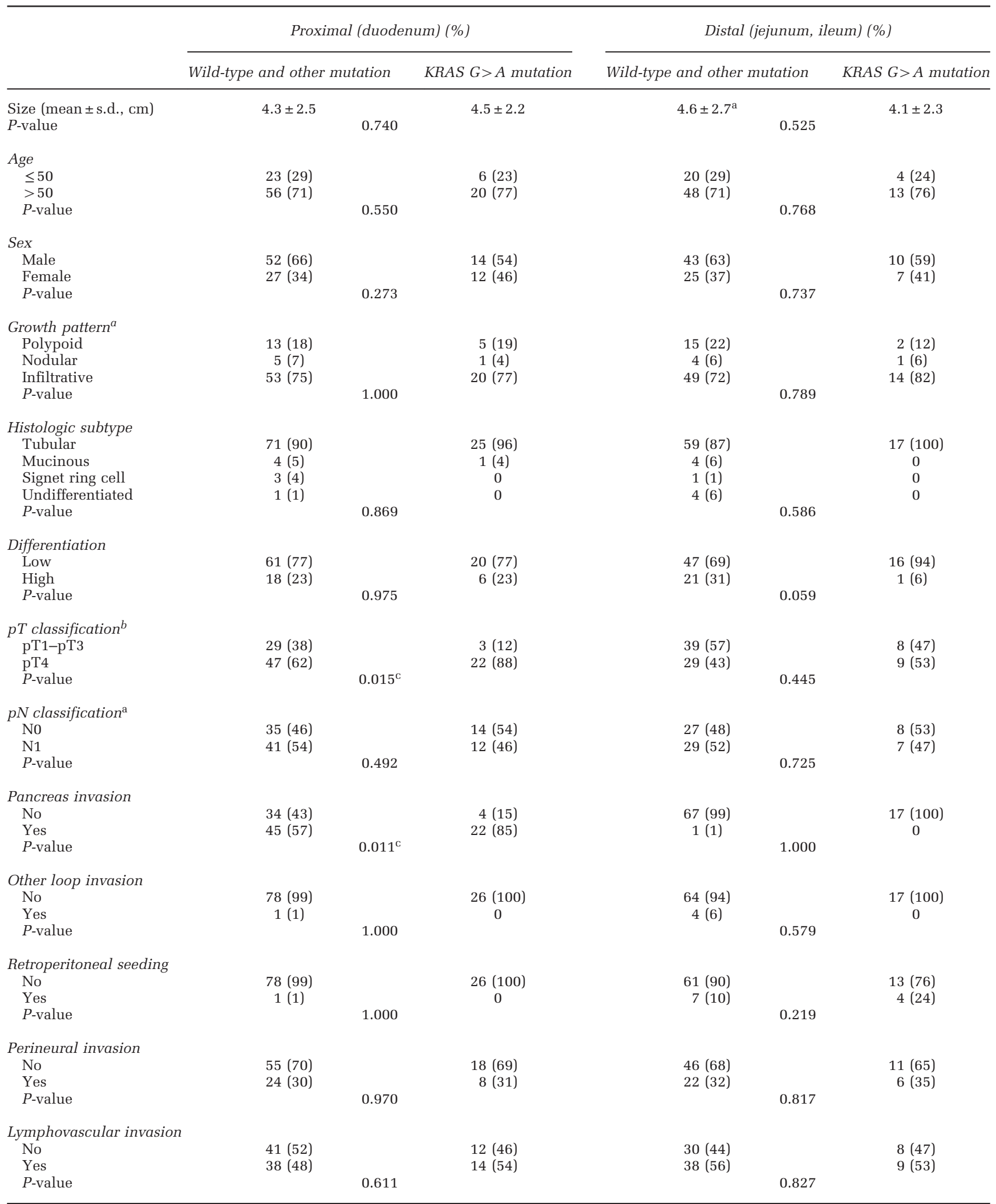

Abbreviation: s.d., standard deviation.

${ }^{\mathrm{a} C a l c u l a t e d}$ using only patients with sufficient available data.

$\mathrm{b}_{\text {Excluding patients with pTis. }}$

${ }^{\mathrm{c}}$ Statistically significant $(P<0.05)$. 
Table 5 Correlation between clinicopathologic features and the presence of KRAS G12D mutations according to tumor location

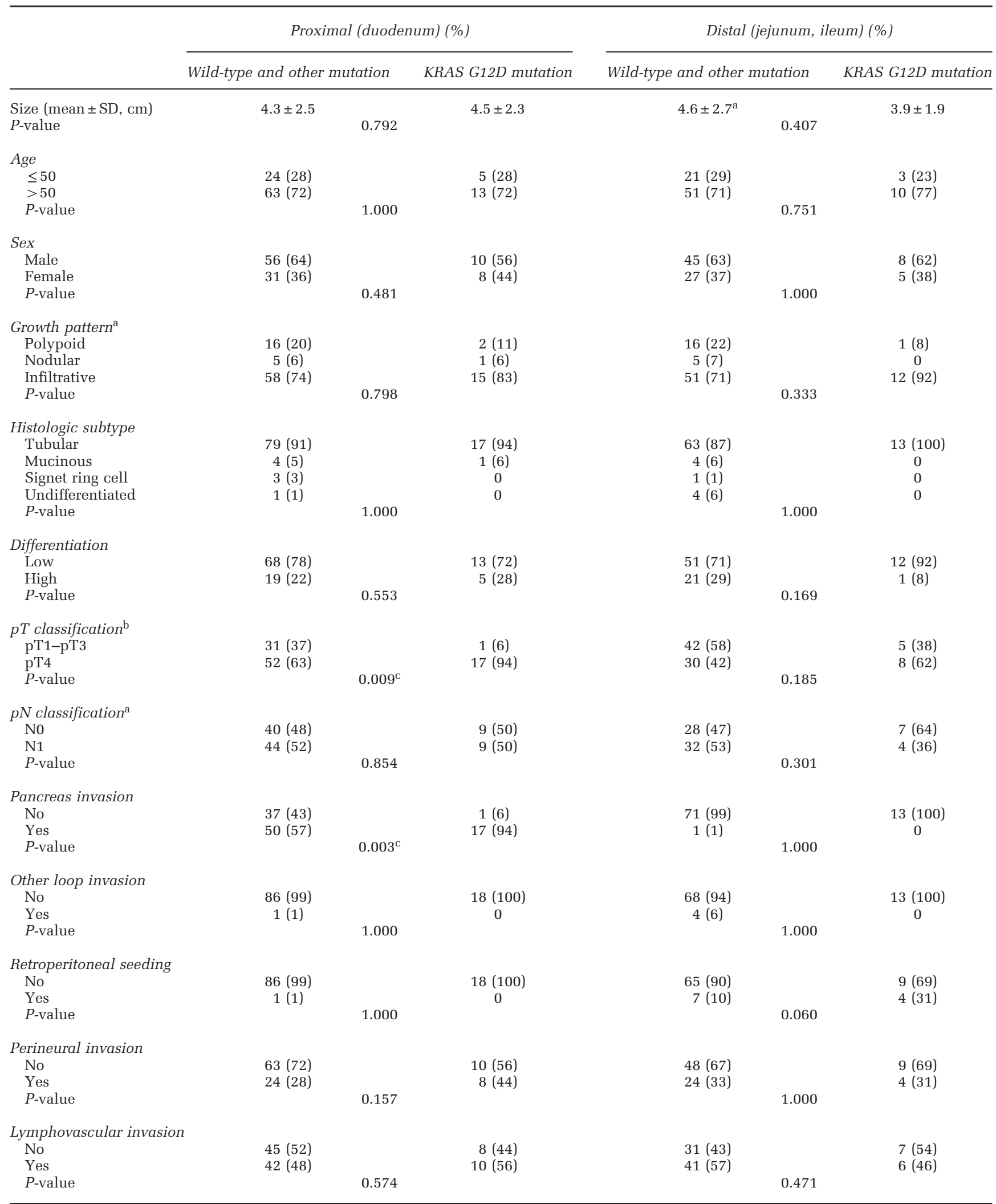

Abbreviation: s.d., standard deviation.

${ }^{\mathrm{a} C a l c u l a t e d}$ using only patients with sufficient available data.

${ }^{b}$ Excluding patients with pTis.

${ }^{\mathrm{c}}$ Statistically significant $(P<0.05)$. 
Table 6 Correlation between clinicopathologic features and the presence of KRAS G>A mutations

\begin{tabular}{|c|c|c|c|c|c|c|}
\hline & \multicolumn{3}{|c|}{ Frequency analysis } & \multicolumn{3}{|c|}{ Multivariate logistic regression analysis } \\
\hline & $\begin{array}{c}\text { Wild-type and } \\
\text { other mutations (\%) }\end{array}$ & $\begin{array}{c}\text { KRAS } G>A \\
\text { mutation }(\%)\end{array}$ & P-value & OR & $95 \% C I$ & $\mathrm{P}$-value \\
\hline \multicolumn{7}{|l|}{ Location } \\
\hline Proximal & $79(54)$ & $26(60)$ & 0.435 & 1.313 & $0.700-2.463$ & 0.396 \\
\hline Distal & $68(46)$ & $17(40)$ & & & & \\
\hline \multicolumn{7}{|c|}{ Differentiation } \\
\hline Low & $108(73)$ & $36(84)$ & 0.167 & 0.747 & $0.474-1.177$ & 0.209 \\
\hline High & $39(27)$ & 7 (16) & & & & \\
\hline \multicolumn{7}{|c|}{$p T$ classification $^{\mathrm{a}}$} \\
\hline pT1-pT3 & $68(47)$ & $11(26)$ & $0.015^{\mathrm{b}}$ & 1.588 & $1.085-2.324$ & $0.017^{\mathrm{b}}$ \\
\hline $\mathrm{pT} 4$ & $76(53)$ & $31(74)$ & & & & \\
\hline \multicolumn{7}{|c|}{ Pancreas invasion } \\
\hline No & $101(69)$ & $21(49)$ & $0.017^{\mathrm{b}}$ & 2.507 & $0.553-11.369$ & 0.234 \\
\hline Yes & $46(31)$ & $22(51)$ & & & & \\
\hline
\end{tabular}

Abbreviations: CI, confidence interval; OR, odds ratio.

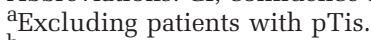

b Statistically significant $(P<0.05)$.

Table 7 Correlation between clinicopathologic features and the presence of KRAS G12D mutations

\begin{tabular}{|c|c|c|c|c|c|c|}
\hline & \multicolumn{3}{|c|}{ Frequency analysis } & \multicolumn{3}{|c|}{$\begin{array}{l}\text { Multivariate logistic } \\
\text { regression analysis }\end{array}$} \\
\hline & $\begin{array}{l}\text { Wild-type and } \\
\text { other mutations (\%) }\end{array}$ & $\begin{array}{l}\text { KRAS G12D } \\
\text { mutation (\%) }\end{array}$ & P-value & OR & $95 \% C I$ & P-value \\
\hline \multicolumn{7}{|l|}{ Location } \\
\hline Proximal & $87(55)$ & $18(58)$ & 0.732 & 0.625 & $0.269-1.453$ & 0.275 \\
\hline Distal & $72(45)$ & $13(42)$ & & & & \\
\hline \multicolumn{7}{|c|}{ Differentiation } \\
\hline Low & $119(75)$ & $25(81)$ & 0.490 & 1.181 & $0.720-1.936$ & 0.510 \\
\hline High & $40(25)$ & $6(19)$ & & & & \\
\hline \multicolumn{7}{|c|}{$p T$ classification ${ }^{\mathrm{a}}$} \\
\hline рT1-pT3 & $73(47)$ & $6(19)$ & $0.004^{\mathrm{b}}$ & 0.519 & $0.324-0.833$ & $0.007^{\mathrm{b}}$ \\
\hline pT4 & $82(53)$ & $25(81)$ & & & & \\
\hline \multicolumn{7}{|c|}{ Pancreas invasion } \\
\hline No & $108(68)$ & $14(45)$ & $0.016^{\mathrm{b}}$ & 0.330 & $0.050-2.175$ & 0.249 \\
\hline Yes & $51(32)$ & $17(55)$ & & & & \\
\hline
\end{tabular}

Abbreviations: CI, confidence interval; OR, odds ratio.

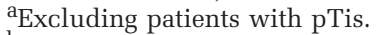

${ }^{\mathrm{b}}$ Statistically significant $(P<0.05)$.

Figure 1 Survival of patients with small intestinal adenocarcinomas based on KRAS or BRAF mutational status. (a) Small intestinal adenocarcinoma patients with KRAS mutations tended to have relatively shorter overall survival outcomes (median, 21.5 months) than those with wild-type KRAS (38.5 months), but this effect does not reach statistical significance $(P=0.116$, log-rank test). (b) Small intestinal adenocarcinoma patients having either KRAS or BRAF mutation (median, 22.6 months) also tended to have shorter survival times than those with wild-type $K R A S$ and $B R A F$ (38.5 months; $P=0.148$ ). There were no significant differences in the survival time distributions of (c) patients with KRAS codon 12 mutations (median, 21.0 months) and those with codon 13 mutations (21.5 months; $P=0.805)$, (d) patients with KRAS G > A mutations (median, 21.0 months) and those with KRAS wild-type or mutations other than $\mathrm{G}>\mathrm{A}$ (36.5 months; $P=0.398$ ), and (e) patients with KRAS G12D (median, 17.3 months) and KRAS wild-type patients and those with mutations other than G12D (30.7 months; $P=0.507$ ). 
multivariate logistic regression analysis including location, differentiation, $\mathrm{T}$ classification and pancreatic invasion, KRAS G12D mutation remained
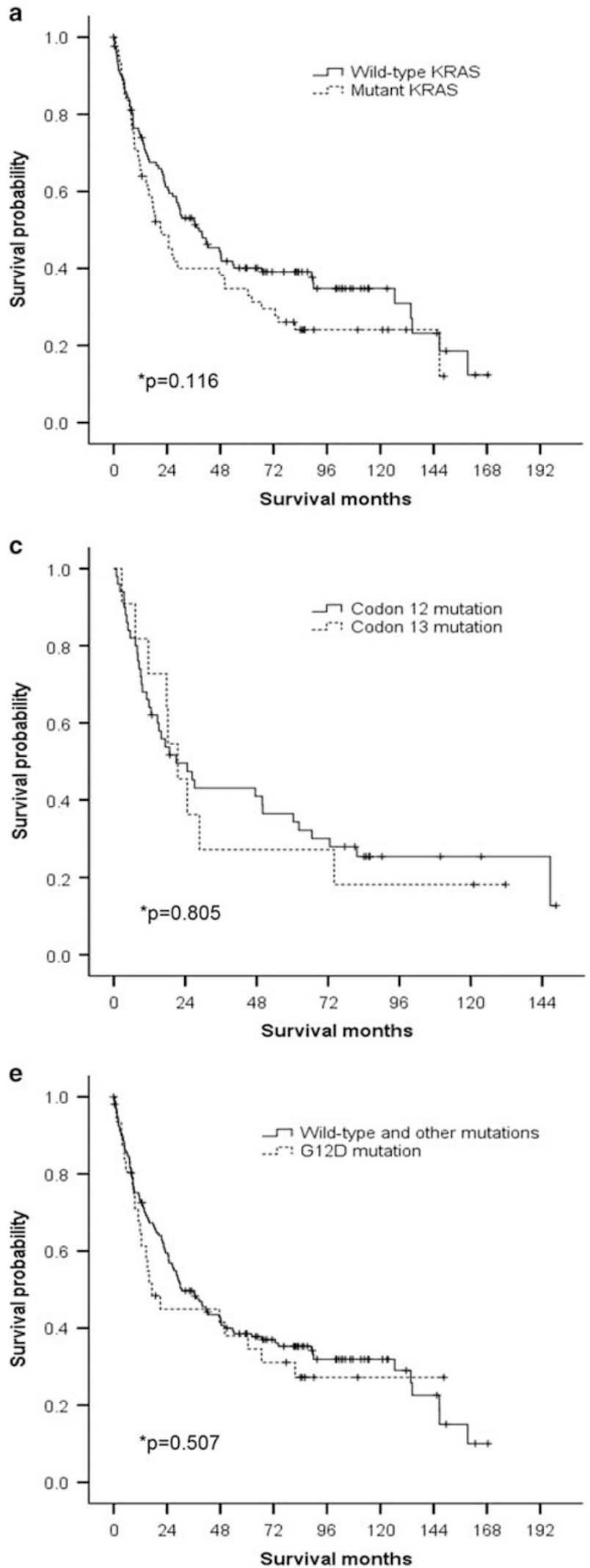

Figure 1 For caption see page 409. significantly more frequent in adenocarcinomas with advanced $\mathrm{T}$ classification (odds ratio $=0.519,95 \%$ CI: $0.324-0.833 ; P=0.007)$.
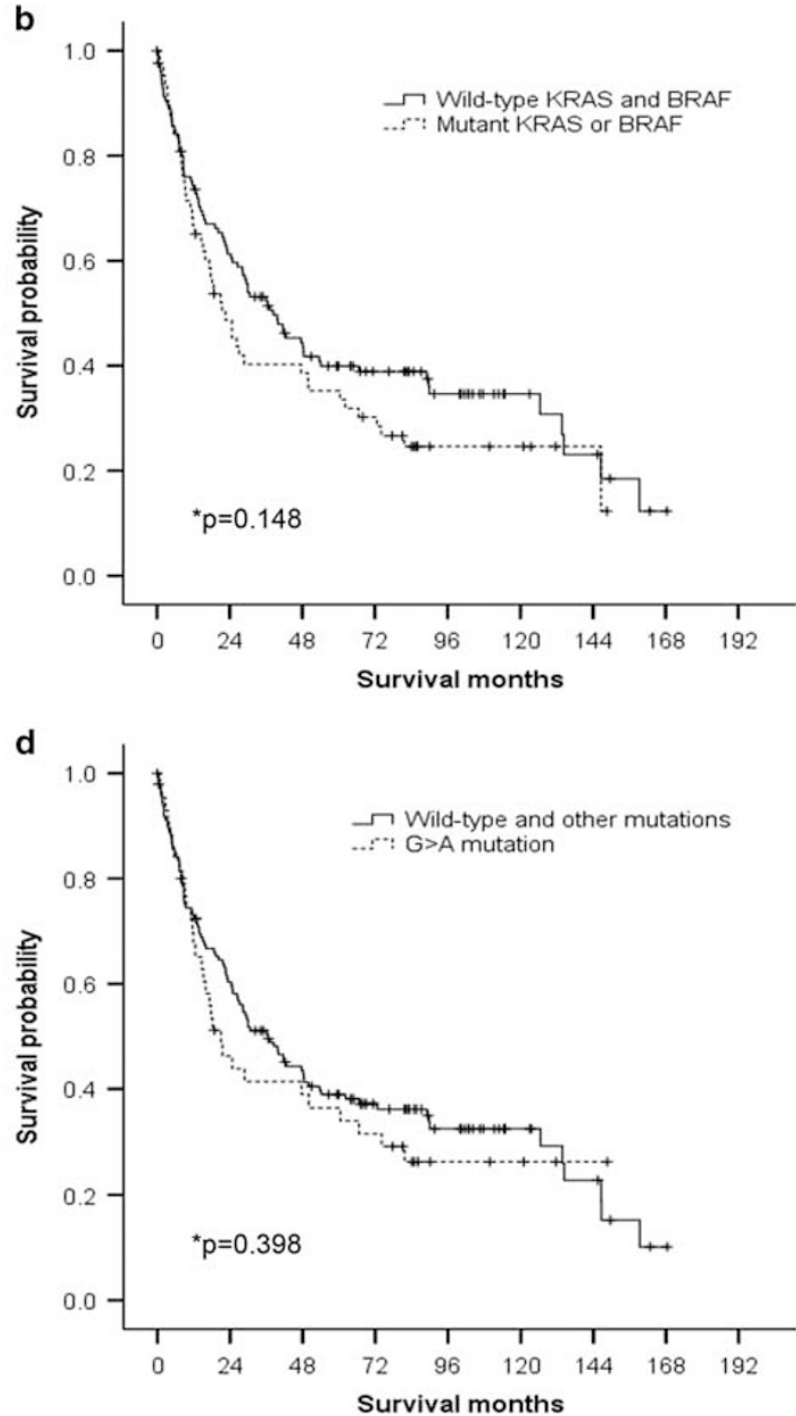

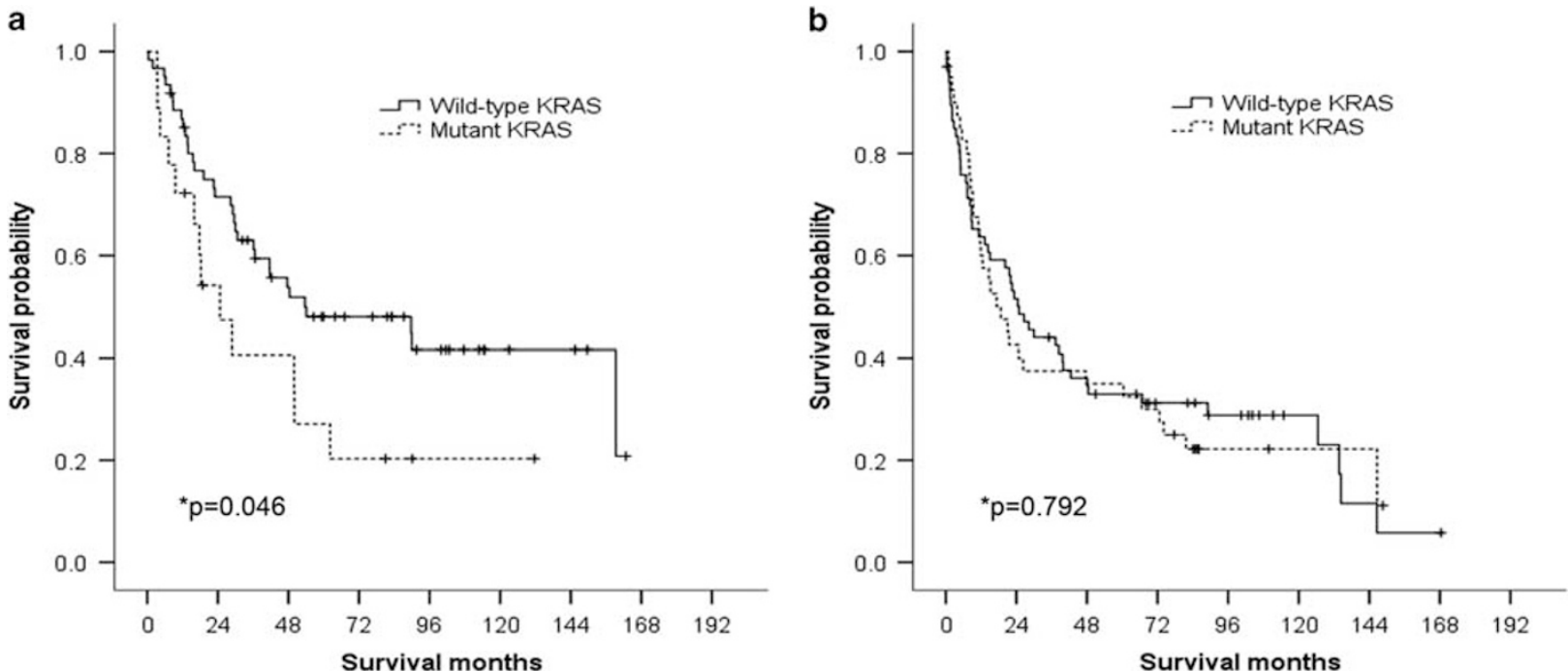

Figure 2 Survival of small intestinal adenocarcinomas patients with KRAS mutation based on $\mathrm{T}$ classification. (a) In lower pT classification (pT1-pT3) subgroup, the survival time for patients with KRAS mutation (median, 46.0 months) was significantly shorter than those with wild-type KRAS (85.4 months; $P=0.046$, log-rank test). (b) In higher pT classification subgroup (pT4), there was no significant difference in survival time distribution between the patients with KRAS mutations (median, 50.1 months) and those with wild-type KRAS (54.8 months; $P=0.792)$.

\section{Survival Analysis}

Small intestinal adenocarcinoma patients with KRAS mutations (median survival time, 21.5 months) had shorter overall survival outcomes than those with wild-type $K R A S$, but the difference did not reach statistical significance (38.5 months; $P=0.116$, logrank test; Figure 1a). The survival times of the two $B R A F$ mutation carriers were also relatively short $(P=0.675)$. There were no significant differences in survival time distribution between the patients with KRAS or BRAF mutations (median, 22.6 months) and those with wild-type KRAS and BRAF (38.5 months; $P=0.148$; Figure $1 b$ ).

When overall survival times were compared based on mutational status, the median survival time of the patients with codon 12 KRAS mutations was not significantly different (21.0 months) from those with codon 13 mutations (21.5 months; $P=0.805$; Figure 1c). Small intestinal adenocarcinoma patients with KRAS G>A mutations were associated with shorter survival time (median, 21.0 months) than those with KRAS wild-type and those with other than $\mathrm{G}>\mathrm{A}$ mutations (36.5 months), but this effect did not reach statistical significance $(P=0.398$; Figure 1d). There were no significant differences in survival time distributions between carriers with KRAS G12D (median, 17.3 months) and KRAS wildtype patients and those with mutations other than G12D (30.7 months; $P=0.507$; Figure 1e).

In terms of tumor location, no relation between KRAS mutation and overall survival was seen in proximal versus distal adenocarcinomas. In addition, KRAS mutation subtype was also not related to the patient survival in either proximal or distal adenocarcinomas.
Table 8 Multivariate analysis of the small intestinal adenocarcinoma patients with lower T classifications (pT1-pT3)

\begin{tabular}{lcccc}
\hline & & \multicolumn{2}{c}{$95 \%$ CI } & \\
\cline { 3 - 4 } & & & \\
& Relative risk & Lower & Upper & P-value \\
\hline KRAS mutation & 2.817 & 1.360 & 5.833 & $0.005^{\mathrm{a}}$ \\
Age $(\leq 50$ vs $>50)$ & 1.835 & 0.897 & 3.756 & 0.097 \\
Sex & 1.789 & 0.905 & 3.535 & 0.094 \\
Location & 1.134 & 0.804 & 1.600 & 0.473 \\
Differentiation & 1.062 & 0.714 & 1.580 & 0.768 \\
pN classification & 1.793 & 0.768 & 4.185 & 0.177 \\
Retroperitoneal & 0.950 & 0.106 & 8.540 & 0.963 \\
seeding & & & & \\
Perineural invasion & 1.266 & 0.547 & 2.927 & 0.582 \\
Lymphovascular & 4.142 & 2.044 & 8.394 & $<0.0001^{\mathrm{a}}$ \\
invasion & & & & \\
\hline
\end{tabular}

Abbreviation: CI, confidence interval.

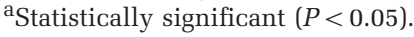

When survival of small intestinal adenocarcinoma patients was compared depending on $\mathrm{T}$ classifications, significant survival differences were observed. In lower $\mathrm{T}$ classification (pT1-pT3) group, the survival time for patients with KRAS mutation (median, 46.0 months) was significantly shorter than those with wild-type KRAS (85.4 months; $P=0.046$; Figure 2a). However, in higher T classification (pT4) group, there was no significant difference in survival time distribution between the patients with KRAS mutations (median, 50.1 months) and those with wild-type KRAS (54.8 months; $P=0.792$; Figure 2b).

After grouping according to tumor location, we also compared the survival of the patients. In the proximal adenocarcinomas, KRAS mutation was not 
Table 9 Previous studies of $K R A S$ and/or $B R A F$ mutations in small intestinal adenocarcinoma

\begin{tabular}{|c|c|c|c|c|c|c|}
\hline \multirow[b]{2}{*}{ Reference } & \multirow[b]{2}{*}{ Country } & \multirow[b]{2}{*}{ Tumors analyzed (n) } & \multicolumn{2}{|c|}{ KRAS mutation } & \multicolumn{2}{|c|}{ BRAF mutation } \\
\hline & & & $\begin{array}{c}\text { Mutation site or } \\
\text { codons }\end{array}$ & $\mathrm{n}(\%)$ & $\begin{array}{c}\text { Mutation site or } \\
\text { codons }\end{array}$ & $\mathrm{n}(\%)$ \\
\hline Laforest et al ${ }^{1}$ & France, Germany & 83: D (39), J (28), I (16) & NS & NS (43) & NS & NS (6) \\
\hline Aparicio et $a l^{3}$ & France & 63: D (32), J (18), I (13) & $12,13,61,146$ & $21 / 49(43)$ & V600E & $1 / 40(3)$ \\
\hline $\mathrm{Fu}$ et $a l^{10}$ & USA & 78: all D & 12,13 & $27 / 78(35)$ & & \\
\hline Warth et al ${ }^{11}$ & Germany & 37: D (15), J (7), I (7), J/I (8) & NS & $11 / 36(31)$ & V600E, etc. ${ }^{\mathrm{a}}$ & $5 / 36(14)^{\mathrm{a}}$ \\
\hline Blaker et $a l^{12}$ & Germany & 21: D (3), J (2), I (6), J/I (10) & Exon 1 & $12 / 21(57)$ & Exons 11, 15 & $1 / 21(5)$ \\
\hline Mitomi et al ${ }^{13}$ & Japan & $7: J(5), I(2)$ & Exon 1 & $5 / 7(71)$ & & \\
\hline Nishiyama et al ${ }^{14}$ & Japan & 35: D (12), J/I (23) & 12 & $2 / 22(9)$ & & \\
\hline Muneyuki et al ${ }^{15}$ & Japan & 20: D (10), J (6), I (4) & $12,13,61$ & $5 / 20(25)$ & & \\
\hline Achille et al ${ }^{16}$ & Italy & 12: all D & 12 & $5 / 12(42)$ & & \\
\hline Rashid et $a l^{17}$ & USA & 23: D (6), D/J (2), J (8), I (7) & $12,13,61$ & $9 / 23(39)$ & & \\
\hline Younes et al ${ }^{18}$ & USA & 28: D (12), J (11), I (5) & $12,13,61$ & $4 / 28(14)$ & & \\
\hline Arai et $a l^{19}$ & Japan & 15: D (2), J (12), I (1) & $12,13,61$ & $8 / 15(53)$ & & \\
\hline Sutter et $a l^{20}$ & USA & 8: D (6), I (2) & 12 & $5 / 6(83)$ & & \\
\hline
\end{tabular}

Abbreviations: D, duodenum; J, jejunum; I, ileum; NS, not stated.

${ }^{\mathrm{a}} \mathrm{V} 600 \mathrm{E}(n=4)$ and a 3 -bp deletion (c1869-1871; $\left.n=1\right)$.

related to the patient survival in either lower or higher pT classification. However, in the distal adenocarcinomas, the patients with KRAS mutations (median, 33.0 months) had significantly shorter overall survival outcomes than those with wildtype KRAS (78.7 months; $P=0.026)$ in a lower pT classification group. On the contrary, in higher $\mathrm{pT}$ classification group of distal adenocarcinomas, the patients with KRAS mutation (median, 30.1 months) had slightly longer survival time than those with wild-type KRAS (22.1 months), but it was not statistically significant $(P=0.428)$.

\section{Prognostic Significance of KRAS Mutation in Lower pT Classification Tumors}

The independent prognostic significance of KRAS mutation and other clinicopathologic factors in small intestinal adenocarcinomas of lower pT classification, which were considered significant by univariate analyses, was further evaluated using the Cox proportional hazards modeling (Table 8). According to this multivariate analysis, KRAS mutation $(P=0.005)$ and the presence of lymphovascular invasion $(P<0.0001)$ are poor independent prognostic predictors of overall survival in small intestinal adenocarcinoma patients with lower pT classification.

\section{Discussion}

We have sequenced the KRAS and BRAF genes of a large number of patients $(n=190)$ in this study and found KRAS mutations in 32\% (61 cases). Although a few previous studies of small intestinal adenocarcinomas have examined KRAS and/or BRAF mutations, which are well-known oncogenes in colorectal cancer, most of these analyses were performed on small numbers of patients (Table 9). ${ }^{1,3,10-20}$ The frequency of KRAS mutations among small intestinal adenocarcinomas ranged from 9 to $83 \%$ in the previous studies. Previous studies from Japan with homogeneous ethnic groups showed a wide range of frequencies of KRAS mutation (9-71\%), ${ }^{13-15,19}$ and other studies with heterogeneous ethnic groups, such as studies from USA, also reported various frequencies of KRAS mutation (14-83\%). ${ }^{10,17,18,20}$ Various KRAS mutation frequencies in the previous studies may be related with different detection methods rather than diverse ethnic background. Several different techniques for detecting KRAS and/or BRAF mutation were used, including restriction fragment length polymorphism, allele-specific oligonucleotide hybridization, PCR-single-strand conformation polymorphism, allele discrimination assay and Sanger sequencing. ${ }^{1,3,10-20}$ In addition to different detection techniques, differences in sample size may contribute to the wide range of KRAS mutation frequencies observed. The overall prevalence of KRAS mutations in the previous studies was $38 \%(150 / 400)$. In those studies with $>35$ KRAS mutations tested, ${ }^{3,10,11}$ the prevalence of KRAS mutation was reported to be between 31 and $43 \%$. This is similar to the prevalence of KRAS mutations in this study. In addition, the prevalence of KRAS mutations among small intestinal adenocarcinomas in the previous studies was similar to that among colorectal cancers. ${ }^{3,6}$

On the other hand, BRAF mutations occur at a lower rate $(0-13 \%)$ than KRAS mutations in colorectal cancers. ${ }^{23}$ BRAF mutations are also rarely encountered among small intestinal adenocarcinomas, where the prevalence of $B R A F$ mutation ranges from 3 to $14 \% .^{1,3,11,12}$ In this study, BRAF mutations were observed in only $1 \%$ of the small intestinal adenocarcinomas. In accord with previous reports for colorectal cancers ${ }^{23}$ and small intestinal adenocarcinomas, ${ }^{1,3,12}$ KRAS and BRAF mutations 
were mutually exclusive ways in our observation as the same with results of the previous studies. ${ }^{3,12}$ Although one study reported concurrent KRAS and $B R A F$ mutation in squamous cell carcinoma of the lung, ${ }^{24}$ to the best our knowledge, there is no report of concurrent KRAS and BRAF mutation in small intestinal adenocarcinomas. Further studies with larger number of cases are needed for identifying the existence of concurrent KRAS and BRAF mutations in small intestinal adenocarcinomas. In summary, the molecular alterations in small intestinal adenocarcinomas are close to those in colorectal cancers, with a high frequency of KRAS mutations and infrequent $B R A F$ mutations.

Several retrospective studies have indicated that chemotherapy prolongs overall survival in patients with advanced small intestinal adenocarcinomas, but there is no standard frontline regimen owing to a lack of randomized trials. ${ }^{5,25}$ Patients with advanced small intestinal adenocarcinomas are often treated with the same chemotherapy regimens as patients with advanced colorectal cancers or gastric cancers, especially 5-fluorouracil (FU) or 5-FU-based schedules. $^{9}$ The combination of 5-FU and a platinum-based agent has been considered more effective than other regimens such as oxaliplatinbased chemotherapy. ${ }^{25,26}$ The main challenge for the near future is to identify molecular markers involved in small bowel carcinogenesis that predict chemosensitivity, and thus to improve survival. ${ }^{5}$ Overman et $a 2^{27}$ observed that a high proportion of small intestinal adenocarcinomas express both EGFR and vascular endothelial growth factor (VEGF), suggesting that these patients may benefit from therapeutic strategies targeting EGFR and VEGF. Targeted therapies such as monoclonal antibodies against VEGF or EGFR combined with chemotherapy have already exhibited significant efficacy in metastatic colorectal cancers. ${ }^{5}$ Given the similar prevalence of KRAS mutations in small intestinal adenocarcinomas and colorectal cancers, we think that targeted therapy with anti-EGFR monoclonal antibody would be particularly appropriate for small intestinal adenocarcinoma patients with wild-type KRAS and $B R A F$ as it is for colorectal cancer patients.

Different genetic mutations may be responsible for different biological effects. In the literature on colorectal cancer, codon 12 mutations of the KRAS are associated with a mucinous phenotype. ${ }^{23}$ By contrast, codon 13 mutations tend to be nonmucinous and are characterized as more aggressive with a greater metastatic potential. ${ }^{23}$ In this study, we did not find any difference in clinicopathologic findings in terms of mucinous/nonmucinous histologic subtype with respect to codon $12 / 13$ mutations of KRAS among the small intestinal adenocarcinomas.

Patients with KRAS and/or BRAF mutations had shorter survival than those with wild-type genes, but the effect was not statistically significant. There has been considerable controversy regarding the prognostic significance of $K R A S / B R A F$ mutations. ${ }^{3,10}$
Many studies have investigated the prognostic value of KRAS/BRAF mutations on retrospectively collected cohorts of colorectal cancers patients, with conflicting results. ${ }^{23,28-32}$ Initially, KRAS was found to be an important prognostic indicator, but this finding was later restricted to $\mathrm{G} 12 \mathrm{~V}$ mutations. ${ }^{31}$ Colorectal cancer patients with $\mathrm{G}>\mathrm{A}$ and $\mathrm{G}>\mathrm{C}$ mutations tended to have a worse prognosis than those with $\mathrm{G}>\mathrm{T}$ mutations. ${ }^{30}$ In addition, mutation of $\mathrm{G}>\mathrm{T}$ but not $\mathrm{G}>\mathrm{A}$ or $\mathrm{G}>\mathrm{C}$ increased the risk of recurrence and death of colorectal cancer patients. ${ }^{32}$ To the best of our knowledge, there have been only two previously reported studies of small intestinal adenocarcinomas concerning the prognostic significance of KRAS mutations in Western populations, ${ }^{3,10}$ and no reports about the prognostic significance of $B R A F$ mutations. Aparicio et $a l^{3}$ reported that mutated KRAS status in small intestinal adenocarcinoma was an independent predictor of longer overall survival, but this was meaningful only in stage IV patients. Whereas about a third of study population $(20 / 63,32 \%)$ was stage IV patients in the study by Aparicio et al, no case of stage IV was present in our study. Fu et al ${ }^{10}$ proposed that KRAS G $>$ A mutation was significantly correlated with late disease stage and poor tumor differentiation, and carriers had an increased risk of distant metastasis and relapse, as well as significantly shorter overall survival. However, in their study, only duodenal adenocarcinomas were included. In this study, KRAS G $>$ A mutation as well as G12D mutation was closely related to advanced $\mathrm{T}$ classification, as in the study of $\mathrm{Fu}$ et al.

In addition, we found that mutated KRAS status was an independent predictor of poor survival in small intestinal adenocarcinoma, particularly in patients of lower pT classification. In the literature about colorectal cancers, KRAS mutations have been detected in the earliest neoplastic lesions found in colonic mucosa, and appear to exert a strong influence on the growth of polyps and early cancers. Nash et $a l^{33}$ reported that the patients with mutant KRAS and microsatellite stability had significantly worse survival than other groups among the earlystage colorectal cancer (stages I and II). To the best of our knowledge, this study is the first report to identify the prognostic significance of KRAS mutations in lower pT classification of small intestinal adenocarcinomas.

Codons 61 and 146 mutation are additional hotspots for KRAS mutations, and data from a small number of studies of colorectal cancers suggest that mutation at these sites predicts resistance to antiEGFR therapy. ${ }^{34}$ Despite their growing clinical relevance, the clinicopathological and molecular features of colorectal cancers with KRAS codon 61 or 146 mutations remain largely unknown. A few studies have encountered mutations of codons 61 and 146 of KRAS in small intestinal adenocarcinomas. ${ }^{3,15,17-19}$ Arai et al ${ }^{19}$ described only one case of a codon 61 KRAS mutation among eight cases of mutant KRAS genes in small intestinal adenocarcinomas. Aparicio 
et $a l^{3}$ only documented KRAS codon $61(n=2)$ and codon $146(n=1)$ mutations in a total of 21 cases of mutated KRAS. As we only examined codons 12 and 13 KRAS mutations, we do not have information regarding the frequency of KRAS mutations of codons 61 and 146. Therefore, further studies are needed to define the predictive value of these mutations in small intestinal adenocarcinomas.

In conclusion, our data from 190 small intestinal adenocarcinoma patients demonstrate that KRAS and, infrequently, $B R A F$ mutations are observed in a subset of small intestinal adenocarcinomas. KRAS mutations are associated with higher pT classification and pancreas invasion. Small intestinal adenocarcinoma patients with either KRAS or BRAF mutation have a tendency toward shorter survival than those with wild-type KRAS. Mutation of KRAS oncogene is a worse prognostic predictor in small intestinal adenocarcinomas of lower pT classification. Our observations suggest that targeted therapies such as anti-EGFR chemotherapy could be beneficial in the two-thirds of small intestinal adenocarcinoma patients with wild-type KRAS and BRAF if they have metastatic disease.

\section{Acknowledgments}

This research was supported by the Basic Science Research Program through the National Research Foundation of Korea (NRF) funded by the Ministry of Education, Science and Technology (2010-0004807) and a grant (2013-554) from the Asan Institute for Life Sciences, Seoul, Republic of Korea.

\section{Disclosure/conflict of interest}

The authors declare no conflict of interest.

\section{References}

1 Laforest A, Aparicio T, Zaanan A et al. ERBB2 gene as a potential therapeutic target in small bowel adenocarcinoma. Eur J Cancer 2014;50:1740-1746.

2 Siegel R, Ma J, Zou Z et al. Cancer statistics, 2014. CA Cancer J Clin 2014;64:9-29.

3 Aparicio T, Svrcek M, Zaanan A et al. Small bowel adenocarcinoma phenotyping, a clinicobiological prognostic study. Br J Cancer 2013;109:3057-3066.

4 Chang $\mathrm{HK}$, Yu E, Kim J et al. Adenocarcinoma of the small intestine: a multi-institutional study of 197 surgically resected cases. Hum Pathol 2010;41: 1087-1096.

5 Zaanan A, Gauthier M, Malka D et al. Second-line chemotherapy with fluorouracil, leucovorin, and irinotecan (FOLFIRI regimen) in patients with advanced small bowel adenocarcinoma after failure of first-line platinum-based chemotherapy: a multicenter AGEO study. Cancer 2011;117:1422-1428.

6 Italiano A, Hostein I, Soubeyran I et al. KRAS and BRAF mutational status in primary colorectal tumors and related metastatic sites: biological and clinical implications. Ann Surg Oncol 2010;17:1429-1434.

7 Santini D, Fratto ME, Spoto C et al. Cetuximab in small bowel adenocarcinoma: a new friend? Br J Cancer 2010;103:1305 author reply 6.

8 De Dosso S, Molinari F, Martin V et al. Molecular characterisation and cetuximab-based treatment in a patient with refractory small bowel adenocarcinoma. Gut 2010;59:1587-1588.

9 Raghav K, Overman MJ. Small bowel adenocarcinomas-existing evidence and evolving paradigms. Nat Rev Clin Oncol 2013;10:534-544.

$10 \mathrm{Fu} \mathrm{T}$, Guzzetta AA, Jeschke J et al. KRAS G>A mutation favors poor tumor differentiation but may not be associated with prognosis in patients with curatively resected duodenal adenocarcinoma. Int J Cancer 2013;132:2502-2509.

11 Warth A, Kloor M, Schirmacher P et al. Genetics and epigenetics of small bowel adenocarcinoma: the interactions of CIN, MSI, and CIMP. Mod Pathol 2011;24: 564-570.

12 Blaker H, Helmchen B, Bonisch A et al. Mutational activation of the RAS-RAF-MAPK and the Wnt pathway in small intestinal adenocarcinomas. Scand J Gastroenterol 2004;39:748-753.

13 Mitomi H, Nakamura T, Ihara A et al. Frequent Ki-ras mutations and transforming growth factor-alpha expression in adenocarcinomas of the small intestine: report of 7 cases. Dig Dis Sci 2003;48:203-209.

14 Nishiyama K, Yao T, Yonemasu H et al. Overexpression of p53 protein and point mutation of K-ras genes in primary carcinoma of the small intestine. Oncol Rep 2002;9:293-300.

15 Muneyuki T, Watanabe M, Yamanaka M et al. Combination analysis of genetic alterations and cell proliferation in small intestinal carcinomas. Dig Dis Sci 2000;45:2022-2028.

16 Achille A, Baron A, Zamboni G et al. Molecular pathogenesis of sporadic duodenal cancer. Br J Cancer 1998;77:760-765.

17 Rashid A, Hamilton SR. Genetic alterations in sporadic and Crohn's-associated adenocarcinomas of the small intestine. Gastroenterology 1997;113:127-135.

18 Younes N, Fulton N, Tanaka R et al. The presence of $\mathrm{K}-12$ ras mutations in duodenal adenocarcinomas and the absence of ras mutations in other small bowel adenocarcinomas and carcinoid tumors. Cancer 1997;79:1804-1808.

19 Arai M, Shimizu S, Imai Y et al. Mutations of the Ki-ras, p53 and APC genes in adenocarcinomas of the human small intestine. Int J Cancer 1997;70:390-395.

20 Sutter T, Arber N, Moss SF et al. Frequent K-ras mutations in small bowel adenocarcinomas. Dig Dis Sci 1996;41:115-118.

21 Jun SY, Eom DW, Park H et al. Prognostic significance of CDX2 and mucin expression in small intestinal adenocarcinoma. Mod Pathol 2014;27:1364-1374.

22 Hamiton SR, Nakamura S-I, Bosman FT et al. Carcinoma of the colon and rectumIn:Bosman FT, Carneiro $\mathrm{F}$, Hruban RH et al.(eds) WHO Classification of Tumours of the Digestive System4th edn.IARC: Lyon, 2010, pp 134-146.

23 Heinemann V, Stintzing S, Kirchner T et al. Clinical relevance of EGFR- and KRAS-status in colorectal cancer patients treated with monoclonal antibodies directed against the EGFR. Cancer Treat Rev 2009;35: $262-271$. 
24 Li B, Lu JC, He D et al. Rapid onset lung squamous cell carcinoma with prominent peritoneal carcinomatosis and an eosinophilic leukemoid reaction, with coexistence of the BRAF V600E and oncogenic KRAS G12A mutations: a case report. Oncol Lett 2014;8:589-593.

25 Xiang XJ, Liu YW, Zhang L et al. A phase II study of modified FOLFOX as first-line chemotherapy in advanced small bowel adenocarcinoma. Anticancer Drugs 2012;23:561-566.

26 Zaanan A, Costes L, Gauthier M et al. Chemotherapy of advanced small-bowel adenocarcinoma: a multicenter AGEO study. Ann Oncol 2010;21:1786-1793.

27 Overman MJ, Pozadzides J, Kopetz S et al. Immunophenotype and molecular characterisation of adenocarcinoma of the small intestine. Br J Cancer 2010;102: 144-150.

28 Richman SD, Seymour MT, Chambers P et al. KRAS and BRAF mutations in advanced colorectal cancer are associated with poor prognosis but do not preclude benefit from oxaliplatin or irinotecan: results from the MRC FOCUS trial. J Clin Oncol 2009;27:5931-5937.

29 Roth AD, Tejpar S, Delorenzi M et al. Prognostic role of KRAS and BRAF in stage II and III resected colon cancer: results of the translational study on the PETACC-3, EORTC 40993, SAKK 60-00 trial. J Clin Oncol 2010;28:466-474.

30 Span M, Moerkerk PT, De Goeij AF et al. A detailed analysis of K-ras point mutations in relation to tumor progression and survival in colorectal cancer patients. Int J Cancer 1996;69:241-245.

31 Andreyev HJ, Norman AR, Cunningham D et al. Kirsten ras mutations in patients with colorectal cancer: the 'RASCAL II' study. Br J Cancer 2001;85:692-696.

32 Andreyev HJ, Norman AR, Cunningham D et al. Kirsten ras mutations in patients with colorectal cancer: the multicenter "RASCAL" study. J Natl Cancer Inst 1998;90:675-684.

33 Nash GM, Gimbel M, Cohen AM et al. KRAS mutation and microsatellite instability: two genetic markers of early tumor development that influence the prognosis of colorectal cancer. Ann Surg Oncol 2010;17:416-424.

34 Imamura Y, Lochhead P, Yamauchi M et al. Analyses of clinicopathological, molecular, and prognostic associations of KRAS codon 61 and codon 146 mutations in colorectal cancer: cohort study and literature review. Mol Cancer 2014;13:135. 